\title{
Prognostic Value of Tumor-Infiltrating Lymphocytes in DCIS: A Meta-Analysis
}

\author{
Shuang-Ling Wu \\ the First Affiliated Hospital of China Medical University \\ Xinmiao Yu \\ the First Affiliated Hospital of China Medical University \\ Xiaoyun Mao \\ the First Affiliated Hospital of China Medical University \\ Feng Jin ( $\nabla_{\text {jinfeng@cmu.edu.cn ) }}$ \\ the First Affiliated Hospital of China Medical University
}

\section{Research Article}

Keywords: Tumor infiltrating lymphocytes (TILs), PD-L1, ductal carcinoma in situ (DCIS), recurrence risk, prognosis

Posted Date: October 8th, 2021

DOl: https://doi.org/10.21203/rs.3.rs-951016/v1

License: @ (1) This work is licensed under a Creative Commons Attribution 4.0 International License. Read Full License 


\section{Abstract}

\section{Background}

Tumor infiltrating lymphocytes (TILs) have been demonstrated to be associated with the prognosis of breast ductal carcinoma in situ (DCIS). In this systematic review and meta-analysis, we investigated the role of TILs and TIL subsets in predicting the recurrence risk of DCIS.

\section{Method}

PubMed, Medline, Web of Science, Embase and Cochrane were searched to identify publications investigating the prognostic role of TILs in DCIS. After study screening, data extraction and risk of bias assessment, a meta-analysis was performed to assess the association between TILs (total TILs, CD4+, CD8+, FOXP3+, PD-L1+ TILs) and the risk of DCIS recurrence.

\section{Results}

A pooled analysis indicated that dense stromal TILs in DCIS were associated with a higher recurrence risk ( $\mathrm{HR} 2.11(95 \% \mathrm{Cl} 1.35-$ 3.28)). Subgroup analysis showed that touching TILs (HR 4.73 (95\% Cl 2.28-9.80)) was more favorable than the TIL ratio (HR 1.49 (95\% $\mathrm{Cl} 1.11-1.99)$ ) in estimating DCIS recurrence risk. Moreover, the predictive value of TILs is suitable for patients who are diagnosed with DCIS and then undergo surgery (HR 2.77, (95\% Cl 1.26-6.07)) or surgery accompanied by radiotherapy (HR 2.26, (95\% $\mathrm{Cl}$ 1.29-3.95)), but not for patients who receive comprehensive adjuvant therapies (HR 1.16, (95\% $\mathrm{Cl} 1.35-3.28)$ ). Among subsets of TILs, dense stromal PD-L1+ TILs were valuable in predicting higher recurrence risk of DCIS.

\section{Conclusion}

This systematic review and meta-analysis confirmed the predictive value of TILs and stromal PD-L1+ TILs in DCIS and indicated an appropriate assessment method for TILs and an eligible population.

\section{Introduction}

Widespread use of mammographic screening has largely increased the detection rate of breast ductal carcinoma in situ (DCIS), which accounts for $20 \%-25 \%$ of newly diagnosed breast cancer [1]. Theoretically, surgical dissection is adequate for DCIS treatment. For patients receiving surgery alone, the local recurrence risk ranges from 10.5-18\% [2], and invasive cancer events occur in $19.2 \%$ of high-grade DCIS patients [3]. In addition, the mortality risk for patients who experience recurrence is 18 times higher than that for those who do not [4]. Thus, it is crucial to identify risk factors in predicting the recurrence risk of DCIS in order to carry out appropriate management strategies.

DCIS is a heterogeneous disease, and its recurrence is a sophisticated process caused by the coevolution of cancer cells and the immune microenvironment. Cells of the tumor microenvironment mainly include tumor infiltrating lymphocytes (TILs), NK cells, macrophages, dendritic cells and myeloid lineage cells [5]. In recent years, accumulating evidence has suggested that TILs in the immune microenvironment are associated with better prognosis in basal-like and HER2-positive invasive breast cancers [6]. In contrast to invasive breast cancer, the role of total TILs and specific subtypes of TILs in DCIS remain ambiguous.

Tumor-infiltrating lymphocytes are variable in cancer progression and can be used to evaluate tumor immunogenicity. In DCIS, dense TILs are demonstrated to be associated with many clinical factors, including younger age, higher tumor grade, comedo necrosis and molecular subtype [7]. However, it remains ambiguous whether dense TILs in DCIS are associated with aggressive tumor features and tumor recurrence risk. A few previous studies have reported that there was no significant difference between dense and sparse TILs groups in tumor recurrence $[8,9]$. Some other studies recently put forward that dense TILs are associated with higher recurrence risk [10-13]. Moreover, some research proposed that the value of TILs in predicting recurrence risk is associated with TIL assessment methods [14] and included patient therapy strategies [15].

Along with total TILs, different subsets of TILs also exhibit diverse functions in cancer progression. The TIL compartment in DCIS is generally composed of $C D 3+T$ cells, followed by $C D 4+T$ cells, $C D 8+T$ cells, $C D 20+B$ cells and FOXP3+ regulatory $T$ cells (Tregs) [16]. Among these cells, it is now believed that CD8+ and CD4+ T cells are involved in the effective immune response, and FOXP3+ regulatory T cells (Tregs) are associated with the suppression of antitumor immunity [17]. However, the exact prognostic role of each 
subset of lymphocytes in DCIS has not yet been clarified. In addition to the above subsets, the expression of PD-L1 in immune cells is also associated with DCIS subtypes and their recurrence [18]. Considering that the PD-1/PD-L1 axis is effective in triple-negative invasive breast cancer immunotherapy [19], the prognostic and therapeutic value of PD-L1 in DCIS remains to be further investigated.

In order to illustrate whether TILs have prognostic value in DCIS, we performed a systematic review and meta-analysis to investigate the prognostic roles of TILs and CD4+, CD8+, FOXP3+ and PD-L1 TIL subtypes in DCIS. We aimed to identify effective clinical characteristics of DCIS in predicting patient recurrence risk.

\section{Method}

The present systematic review and meta-analysis were performed in accordance with the Preferred Reporting Item for Systematic Reviews and Meta-Analyses (PRISMA) statement.

\section{Search strategy}

We performed an extensive literature search of electronic databases including Pubmed, Medline, Web of Science, Excerpta Medica Database (Embase) and Cochrane up to 5 April 2021 by two investigators. The search strategy was in line with published articles, and the following determinant domains were used: ("Ductal Carcinoma in Situ" OR "DCIS" OR "Intraductal Carcinoma") AND ("Tumor infiltrating lymphocyte" OR "Infiltrating lymphocyte" OR "Immune" OR "Immune cell" OR "Immunology" OR "TILs" OR "TIL assessment" OR “lymphocyte" OR “CD4" OR "CD8” OR "FOXP3" OR “PD-L1") AND ("Prognosis" OR "Survival” OR "recurrence”). In addition, All the proceedings in scientific meetings and references of the selected articles were searched to identify associated data. The title and abstract of each study in the search were scanned to exclude clearly irrelevant studies.

\section{Inclusion and exclusion criteria}

Inclusion criteria were as follows: (1). Patients diagnosed with DCIS or DCIS with micro-invasive lesions were pathological examination confirmed; (2). Total TILs and specific subtypes of TILs were measured according to HE and IHC staining; (3). Original research articles and proceedings in English; (4). Correlation of TILs with tumor recurrence was illustrate with Hazard Ratio (HR) and a $95 \%$ confidence interval $(95 \% \mathrm{Cls})$.

Exclusion criteria were as follows: (1). Overlapping articles or repeat analysis; (2). Studies lacking sufficient data for assessing Hazard Ratio (HR) and a $95 \%$ confidence interval (95\% Cls); (3). Types of Case reports, reviews, letters, comments and nonclinical studies; (4). Articles written with non-English.

\section{Data extraction}

All the data from candidate studies were evaluated and extracted by two independent investigators. Disagreements in data extraction were discussed and resolved by consensus. The following data were obtained from each study: year of publication, first author, country of the population studied, pathology of studied samples, total number of included cases, method of TIL's detection, cutoff of dense TIL's classification, cell type of studied TILs, treatment strategy, time of follow-up, deadline (type of recurrence).

\section{Quality assessment}

The Newcastle-Ottawa Scale (NOS) was used to assess the quality of included studies in our meta-analysis. The NOS consists of three items including selection (0-4 points), comparability (0-2 points), and outcome assessment (0-3 points). NOS scored more than 7 were assigned as high-quality studies.

\section{Statical analysis}

The meta-analysis calculated the pooled HR and corresponding $95 \% \mathrm{Cls}$ to evaluate the prognostic value of TILs in DCIS. All statistical analyses were performed with STATA version 15. Higgins I-squared statistic were used to estimate the heterogeneity of the included studies. Heterogeneity analysis was assessed by $P$ and $\mathrm{P}$ heterogeneity, $\mathrm{P}$ heterogeneity $<0.10$ and $P>50 \%$ represented significant heterogeneity. When heterogeneity was observed, the random-effect model was adopted. Sensitivity analysis and metaregression were used to explore the origin of heterogeneity. Publication bias was assessed by Egger test and Begg funnel plot. All statistical tests were two-sided, $P$ value $<0.05$ was considered statistically significant. 


\section{Results}

Literature research

A total of 1039 records were searched in Medline, PubMed, Embase, Cochrane and Web of Science. After excluding duplicates, 619 records remained. Next, we screened the titles and abstracts of the 619 papers, and only 42 papers were included for further full-text review. Among these papers, 20 papers were excluded because they did not provide relevant data in estimating TILs in DCIS, and another 5 were conference abstracts that displayed the same data as other included papers. Next, 2 papers were excluded because they focused on infiltrating macrophage cells and TIL-Bs in DCIS. Ultimately, 15 articles including 17 sets of studies were included for quality assessment. Through quality assessment, 2 articles were excluded because their NOS score are fewer than 7 . Finally, 13 articles including 15 sets of studies were used for following meta-analysis. Among these 13 studies, 12 studies including 14 studies investigated the prognostic role of total TILs in DCIS, and 6 articles containing 10 studies explored the value of TIL subsets in DCIS (Figure 1).

\section{Included studies' characteristics}

Detailed characteristics of the included articles are listed in Table 1. These articles were conducted in the United States (2), China (1), Europe (1), Australia (2), the United Kingdom (3), Singapore (1), the Netherlands (1), Italy (1) and Belgium (1), including approximately 4335 participations. All 13 articles were retrospective cohort studies, 1 of the 13 was a conference abstract, and the others were fullreported articles. Among these, 12 articles, which included 14 sets of studies, evaluated the relationship between TILs and DCIS recurrence, and 6 articles investigated the prognostic value of TIL subtypes (CD4+, CD8+, FOXP3+, PD-L1) in DCIS.

\section{Study quality and risk of bias}

After full-text review, we performed critical assessment for each study by NOS, and the quality of each study is summarized in Figure 2. Most of the studies exhibit excellent quality with more than six stars. Two studies scored fewer than 7 stars due to missing data, unclear TIL assessment method and univariate analysis. Studies with fewer than 7 stars were excluded from our meta-analysis; therefore, 15 studies were eligible for inclusion in the meta-analysis.

\section{The value of total TILs in the recurrence of DCIS}

A total of 14 studies in 12 sets of articles containing 4335 patients were included in our meta-analysis to evaluate the prognostic value of total TILs in DCIS. The results showed that dense TILs in DCIS indicates a higher recurrence risk. The pooled HR was 2.11 $(95 \% \mathrm{Cl}, 1.35-3.28)$ for the total TIL level (dense vs. sparse), with statistically significant heterogeneity $(R=78.3 \%, \mathrm{P}=0.000)($ Figure 3A).

To further investigate the heterogeneity in our meta-analysis, we performed meta-regression and subgroup analyses (Table 2). Through meta-regression analysis, we identified "TIL assessment method" as the main cause of heterogeneity $(P>|t|=0.005)$. Furthermore, in subgroup analysis, we also observed that the assessment method of TILs in different studies may affect the prognostic value of TILs in DCIS. The pooled HR for 11 studies using TIL ratio classification was 1.49 (1.11-1.99), with no obvious heterogeneity between the results of the studies $(R=28.4 \%, P=0.175)$. In the other 3 studies that employed touching-TIL classification, the pooled HR was $4.73(2.28-9.8)$, with slight heterogeneity $(R=72.4 \%, P=0.027)$. The pooled HR indicated that stromal touching TILs in DCIS were associated with recurrence more closely than the stromal TIL ratio (Figure 3B). In addition, we observed that the therapeutic strategy is also significant for the evaluation of the prognostic value of TILs. The pooled HRs for those patients who underwent surgery only or surgery accompanied by radiotherapy were 2.77 (1.26-6.07) and 2.26 (1.29-3.95), respectively. TILs in those patients who experienced comprehensive adjuvant therapy displayed no predictive effect on recurrence risk, with an HR of $1.16(0.62-2.18, R=28.3 \%, P=0.233)$ (Figure $3 C$ ). The prognostic value of TILs in patients receiving diverse therapies varies differently, which indicates that the value of TILs in predicting DCIS recurrence is more suitable for patients diagnosed with DCIS who receive surgery only or surgery accompanied by radiotherapy.

\section{Different subtypes of TILs play different roles in the recurrence of DCIS}

Aside from total TILs, we also investigated the prognostic role of CD4+, CD8+, FOXP3+ and PD-L1+ TILs in DCIS. The pooled HRs of CD4+ and FOXP3+ TILs were estimated to be 1.98, 1.44-3.44 and 1.83, 1.23-2.70, respectively, with no considerable heterogeneity 
between studies (CD4+: $P=45.8 \%, \mathrm{P}=0.174$; FOXP3+: $P=0 \%, \mathrm{P}=0.382$ ). This indicates that dense CD4+, FOXP3+ TILs in DCIS are associated with a higher recurrence risk. In addition to CD4+ and FOXP3+ TILs, we also evaluated the prognostic value of PD-L1+ TILs in DCIS. The pooled HR for stromal PD-L1 TILs was 6.21 (4.26-9.06). Considering that some studies observed positive expression of PD-L1 in intraductal cancer cells in DICS, we further investigated the association between PD-L1+ tumor cells and the recurrence risk of DCIS. The pooled HR for PD-L1+ tumor cells was $3.33(0.65-17.21)$, without apparent heterogeneity $(R=36.8 \%, P=0.206)$. Through the above integrated analysis, we observed that both PD-L1+ TILs and PD-L1+ tumor cells are associated with the recurrence risk of DCIS. Regarding CD8+ TILs, an insufficient number of studies provided data to perform a meta-analysis, and the only study with such data provided showed no significant association between CD8+ TILs and patient recurrence (HR 0.90 (0.47-1.71)) (Figure 4). Taken together, CD4+, FOXP3+, PD-L1 TILs and PD-L1 tumor cells possess the potential to predict the recurrence risk of DCIS, and the stromal PD-L1 is more valuable than the others in evaluating DCIS recurrence risk. Thus, in clinical practice, dense stromal PD-L1+ TILs may act as a favorable marker in predicting higher recurrence risk and poor outcome of DCIS.

\section{Sensitivity analysis}

Sensitivity analysis was performed in our study to estimate the effect of each study on pooled HR by consecutive deletion of each study. The results show that no eligible study exhibited a significant influence on the pooled HR of total TILs. (Figure 5).

\section{Publication bias}

Funnel plots display symmetrical distribution and did not indicate any obvious publication bias affecting the HR for cancer recurrence in the included studies $\left(P_{\text {Begg }}=0.228, P_{\text {Egger }}=0.931\right)$. (Figure 6A-6B).

\section{Discussion}

Some studies have investigated the prognostic role of tumor infiltrating lymphocytes (TILs) in ductal carcinoma in situ (DCIS), however it is still controversial whether total TILs and subtypes of TILs can indicate recurrence risk of DCIS. Therefore, we performed a systematic review and meta-analysis to evaluate the prognostic value of total TILs and some subsets of TILs, including CD4+, CD8+, FOXP3+ and PD-L1, in DCIS.

Our meta-analysis performed integrative analysis of TILs with 14 reports including 4335 patients, revealing that dense stromal TILs in DCIS indicates higher recurrence risk (HR: 2.11, 95\% Cl: 1.35-3.28, $p<0.001, R=78.3 \%$ ). Subgroup analyses indicated that touching TILs is more effective in evaluating patient recurrence-free survival than the stromal TIL ratio. Moreover, it is more suitable to use TILs to evaluate the recurrence risk in patients who undergo surgery or surgery accompanied by radiotherapy than in patients who adopt comprehensive adjuvant therapies after surgery. Subsequent analysis of TIL subsets suggested that PD-L1-positive stomal lymphocytes in DCIS are associated with recurrence risk more closely than the other subset of TILs.

Through comparative analysis, our findings were basically in agreement with the studies of Michael S. Toss et al. [12, 14], Farbod Darvishian et al.[11] and Fei-Fei Xu et al.[13] Some studies did not observe a significant correlation between TILs and tumor recurrence in DCIS[8, 9]. In our observation, the discrepancy was mainly caused by TIL assessment methods and patient therapeutic strategies. Regarding the TIL assessment method, our subgroup indicated that touching TILs were associated with patient recurrence risk more closely than the percentage of stromal TILs, which has been previously pointed out in Michael S. Toss's study [14]. Mechanistically, the immune microenvironment is associated with tumor progression [20]. Touching TILs in the basement membrane may interact with myoepithelial cells and intraductal cancer cells more directly, which may further function in immune suppression and promote invasive progression. Although stromal touching TILs have been proposed to be valuable in predicting the recurrence risk of DCIS in some reports, studies investigating touching TILs in DCIS are still limited, and the effective criterion for touching TILs remains unclear.

Aside from the TIL assessment method, we observed that adjuvant therapies following surgery are also crucial in evaluating TIL predictive value. Recently, Alberto proposed that dense TILs were associated with lower second breast events only in patients who did not receive radiotherapy [15]. In our observation, dense TILs were associated with a higher recurrence risk in patients who received solely surgery or surgery accompanied by radiotherapy. Only in those patients who received comprehensive adjuvant therapies dense 
TILs did not exhibit predictive value in evaluating recurrence risk. The inconsistency between Alberto and our subgroup analysis may be attributed to the diverse proportion of high-risk patients included in each study and different assessment methods for dense TILs. Given that TILs are closely associated with aggressive tumor characteristics, including lower grade, larger tumor size and younger age [7], such patients undergo more adjuvant therapies (radiotherapy or endocrine therapy) to reduce recurrence risk. It has been widely demonstrated that both radiotherapy and endocrine therapy are effective strategies in reducing the recurrence risk of DCIS [21], which may interfere with the predictive value of TILs in DCIS prognosis. Thus, in the future, subgroup analysis of TILs in patients who receive diverse adjuvant treatments will be needed to explore the specific TIL value.

The changes in total TILs and each TIL subset proportion in DCIS may be associated with cancer cell immune states. Therefore, we also performed a meta-analysis for TIL subsets. Considering limited research using IHC staining to investigate subsets of TILs in DCIS, our meta-analysis is restricted. In our study, only CD4+ TILs and FOXP3+ TILs were marginally associated with the recurrence risk of DCIS. In addition to the T-TIL subset study contained in our meta-analysis, some studies also investigated B lymphocytes (TILBs) and tumor-associated macrophages (TAMs), and tried to use the TIL ratio to assess DCIS recurrence risk. In I Miligy's study, CD20+/CD19+ TIL-Bs were demonstrated to be associated with a shorter recurrence-free interval [22]; the prognostic value of CD68+ and CD163+ TAMs were also proposed in Xiao-Yang Chen [23] and Aye Aye Thike's studies [24]. In addition, the ratio of FOXP3+/CD8+ and FOXP3+/CD4+ cells in Milim Kim's study [18], and CD8HLADR+/- and CD115+ cells in Michael J. Campbell's study [25], were associated with recurrence-free survival in DCIS. Although some of the studies observed positive results, related studies are still limited, and there is a lack of uniform and suitable assessment methods for the TIL ratio. Thus, no studies about TIL-B, macrophage and TIL ratios can be included in our meta-analysis. Considering that the PD1/PD-L1 axis plays a key role in T cell-mediated immunity [26] and that some reports have proposed that PD-L1 could evaluate the prognosis of DCIS, we also performed integrated analysis of PD-L1 in DCIS. Although there is still a lack of uniform PD-L1 clones and assessment methods, most of the studies observed that limited cancer cells in DCIS exhibit PD-L1 expression, while PD-L1 staining in immune cells is partly positive [7, 9]. Both PD-L1 staining in immune cells and tumor cells has been investigated in some research, but which kind of PD-L1 expression is valuable in predicting the recurrence risk of DCIS remains unclear. In our subgroup analysis, we observed that stromal PD-L1 in immune cells was associated with recurrence risk more closely than tumor cell PD-L1 staining, and dense stromal PD-L1+ TILs may indicate higher recurrence risk for DCIS in clinical practice. In previous reports, some studies demonstrated that PD-L1 is associated with molecular subtype, and stromal PD-L1 is more prevalent in triple-negative breast cancer [7, 12]. Micheal S. Toss's study also proposed that the association between stromal PD-L1 and recurrence risk for DCIS varies differently in diverse molecular subtypes [12]. In addition, some studies observed that PD-L1 is associated with younger age and TIL density [9]. Therefore, we proposed that the inconsistent value and distribution of PD-L1 in different studies may be explained by the diverse clinical characteristics of the included patients. In the future, the value of PD-L1 among patients with different molecular subtypes and effective clones of PD-L1 in risk assessment remain to be investigated.

Aside from prediction of recurrence risk, it is also important to identify effective markers or tumor characteristics in order to provide accurate guidance of therapies to patients. Clinical trials have widely demonstrated that radiotherapy and endocrine therapy following breast-conserving surgery are effective in reducing the recurrence risk of DCIS [21]. Owing to the side effects and ambiguous survival benefit, many patients suffer through unnecessary adjuvant therapies. Along with high-risk prediction, TILs in DCIS are also valuable in predicting efficiency of adjuvant therapy. Fei-Fei Xu's report indicated that DICS accompanied by dense TILs is less sensitive to radiotherapy, while sparse TILs are more suitable for radiotherapy [13]. Given that few studies focus on the value of TIL in adjuvant therapy guidance, further comparative investigation could not be performed. Therefore, the relationship between TILs/subset of TILs and each kind of adjuvant therapy efficiency still needs further investigation. Similar to traditional treatments, immunotherapy of breast cancer has also achieved promising results in invasive breast cancer [27]. Amid DCIS, some reports have demonstrated that PD-L1-positive lymphocytes are more prevalent in high-grade, triple-negative tumors [7]. In our meta-analysis, we also observed that PD-L1 expression in DCIS was associated with patient recurrence risk. Considering that triple-negative DCIS could not benefit from endocrine therapy, targeting PD-L1 in DCIS clinical treatment may be developed as a potential immunotherapy strategy in the future. However, limited intraductal cancer cells in DCIS express PD-L1, and the PD-1/PD-L1 checkpoint pathway in DCIS remains unclear. Immunotherapy based on PD1/PD-L1 in early breast lesions remains to be explored.

There was obvious heterogeneity across the included studies in HR for recurrence $(p<0.001, P=78.3 \%)$. Through sensitivity analysis and meta-regression, we observed that the heterogeneity was mainly from the assessment method of TILs. Moreover, the main limitation of our study is that the eligible studies were relatively small, and all of the included records were retrospective studies. Our study is a literature-based analysis. We checked the publication bias with both Egger's and Begg's tests and did not observe any 
publication bias. While studies with positive results were more prone to be published, relative data that did not observe a positive correlation between TILs and DCIS recurrence risk were partly unavailable [28, 29]. Moreover, only studies with English language were included, which may also cause potential publication bias.

Taken together, our meta-analysis confirmed the prognostic role of tumor infiltrating lymphocytes (TILs) in DCIS and proposed that the assessment method of TILs and patient adjuvant therapies are critical in evaluating TIL predictive value. In addition, we identified PD-L1 in TIL subsets as a potential marker in predicting the higher recurrence risk of DCIS. To achieve precise prediction of the recurrence risk of DCIS, more studies investigating TILs in a subpopulation of DCIS patients are needed in the future.

\section{Conclusions}

For patients who received surgery or surgery accompanied with radiotherapy, dense stromal TILs in DCIS indicated higher recurrence risk. Among TIL subsets, dense stromal PD-L1+ TILs were valuable in predicting higher recurrence risk of DCIS.

\section{Abbreviations}

TILs: tumor infiltrating lymphocytes; t-TILs: stromal touching TILs; $\mathrm{p}$-TILs: percentage of stromal TILs; TAMs: tumor-associated macrophages; DCIS: ductal carcinoma in situ; p-DCIS: pure DCIS; i-DCIS: pure DCIS mixed with IDC; m-DCIS: pure DCIS mixed with microinvasive cancer; HE: hematoxylin and eosin staining; IHC: immunohistochemistry staining; TC: tumor cell; S: surgery; BCS: breast conserving surgery; MAS: mastectomy; AT: adjuvant therapy; RT: radiation therapy; HT: hormonal therapy; IR: ipsilateral recurrence; AR: all kinds of recurrence; SBE: second breast cancer events; HR: hazard ratio; Cl: confidence intervals; PRISMA: preferred reporting item for systematic reviews and meta-analyses; Embase: excerpta medica database; NOS: the newcastle-ottawa scale; UK: united kingdom; USA: united states of america.

\section{Declarations}

\section{Ethics approval and consent to participate}

Not applicable

\section{Consent for publication}

Not applicable

\section{Availability of data and materials}

Not applicable

\section{Competing interests}

The authors declare that they have no competing interests

\section{Funding}

This work was supported by grants from National Natural Science Foundation of China under Grant 82073282 and 81972791.

\section{Authors' contribution}

SLW and XYM performed data acquisition, SLW contributed to data interpretation, statistical analysis and drafting the manuscript. SLW, XMY, XYM and FJ contributed to the study design and data interpretation. All the authors contributed to the article and approved the manuscript.

\section{Acknowledgements}

Not applicable 


\section{References}

1. Kerlikowske K: Epidemiology of ductal carcinoma in situ. J Natl Cancer Inst Monogr 2010, 2010(41):139-141.

2. Ward EM, DeSantis CE, Lin CC, Kramer JL, Jemal A, Kohler B, Brawley OW, Gansler T: Cancer statistics: Breast cancer in situ. CA Cancer J Clin 2015, 65(6):481-495.

3. Solin LJ, Gray R, Baehner FL, Butler SM, Hughes LL, Yoshizawa C, Cherbavaz DB, Shak S, Page DL, Sledge GW, Jr. et al: A multigene expression assay to predict local recurrence risk for ductal carcinoma in situ of the breast. J Natl Cancer Inst 2013, 105(10):701-710.

4. Narod SA, Iqbal J, Giannakeas V, Sopik V, Sun P: Breast Cancer Mortality After a Diagnosis of Ductal Carcinoma In Situ. JAMA Oncol 2015, 1(7):888-896.

5. Gil Del Alcazar CR, Huh SJ, Ekram MB, Trinh A, Liu LL, Beca F, Zi X, Kwak M, Bergholtz H, Su Y et al: Immune Escape in Breast Cancer During In Situ to Invasive Carcinoma Transition. Cancer Discov 2017, 7(10):1098-1115.

6. Denkert C, von Minckwitz G, Darb-Esfahani S, Lederer B, Heppner BI, Weber KE, Budczies J, Huober J, Klauschen F, Furlanetto J et al: Tumour-infiltrating lymphocytes and prognosis in different subtypes of breast cancer: a pooled analysis of 3771 patients treated with neoadjuvant therapy. Lancet Oncol 2018, 19(1):40-50.

7. Hendry S, Pang JMB, Byrne DJ, Lakhani SR, Cummings MC, Campbell IG, Mann GB, Gorringe KL, Fox SB: Relationship of the Breast Ductal Carcinoma In Situ Immune Microenvironment with Clinicopathological and Genetic Features. Clinical Cancer Research 2017, 23(17):5210-5217.

8. Pruneri G, Lazzeroni M, Bagnardi V, Tiburzio GB, Rotmensz N, DeCensi A, Guerrieri-Gonzaga A, Vingiani A, Curigliano G, Zurrida S et al: The prevalence and clinical relevance of tumor-infiltrating lymphocytes (TILs) in ductal carcinoma in situ of the breast. Ann Oncol 2017, 28(2):321-328.

9. Thompson E, Elwood H, Sharma R, Meeker A, Warzecha H, Argani P, Cimino-Mathews A: The immune microenvironment of breast ductal carcinoma in situ: A pilot study. Laboratory Investigation 2015, 95:70A.

10. Van Bockstal M, Lambein K, Smeets A, Slembrouck L, Neven P, Nevelsteen I, Weltens C, Van Limbergen E, Christiaens MR, Van Ongeval $\mathrm{C}$ et al: Stromal characteristics are adequate prognosticators for recurrence risk in ductal carcinoma in situ of the breast. Ejso 2019, 45(4):550-559.

11. Darvishian F, Ozerdem U, Adams S, Chun J, Pirraglia E, Kaplowitz E, Guth A, Axelrod D, Shapiro R, Price A et al: Tumor-Infiltrating Lymphocytes in a Contemporary Cohort of Women with Ductal Carcinoma In Situ (DCIS). Annals of surgical oncology 2019, 26(10):3337-3343.

12. Toss MS, Abidi A, Lesche D, Joseph C, Mahale S, Saunders H, Kader T, Miligy IM, Green AR, Gorringe KL et al: The prognostic significance of immune microenvironment in breast ductal carcinoma in situ. British journal of cancer 2020, 122(10):1496-1506.

13. Xu F-F, Zheng S-F, Xu C, Cai G, Wang S-B, Qi W-X, Wang C-F, Chen J-Y, Lu C: Prognostic and predictive significance of tumor infiltrating lymphocytes for ductal carcinoma in situ. Oncoimmunology 2021, 10(1):1875637.

14. Toss MS, Miligy I, Al-Kawaz A, Alsleem M, Khout H, Rida PC, Aneja R, Green AR, Ellis IO, Rakha EA: Prognostic significance of tumor-infiltrating lymphocytes in ductal carcinoma in situ of the breast. Modern pathology: an official journal of the United States and Canadian Academy of Pathology, Inc 2018, 31(8):1226-1236.

15. Farolfi A, Petracci E, Serra L, Ravaioli A, Bravaccini S, Ravaioli S, Tumedei MM, Ulivi P, Canale M, Puccetti M et al: TumorInfiltrating Lymphocytes (TILs) and Risk of a Second Breast Event After a Ductal Carcinoma in situ. Front Oncol 2020, 10:1486.

16. Chen X-Y, Yeong J, Thike AA, Bay BH, Tan PH: Prognostic role of immune infiltrates in breast ductal carcinoma in situ. Breast cancer research and treatment 2019, 177(1):17-27.

17. Luen S, Virassamy B, Savas P, Salgado R, Loi S: The genomic landscape of breast cancer and its interaction with host immunity. Breast 2016, 29:241-250.

18. Kim M, Chung YR, Kim HJ, Woo JW, Ahn S, Park SY: Immune microenvironment in ductal carcinoma in situ: a comparison with invasive carcinoma of the breast. Breast Cancer Res 2020, 22(1):32.

19. Bastaki S, Irandoust M, Ahmadi A, Hojjat-Farsangi M, Ambrose P, Hallaj S, Edalati M, Ghalamfarsa G, Azizi G, Yousefi M et al: PDL1/PD-1 axis as a potent therapeutic target in breast cancer. Life Sci 2020, 247:117437.

20. Gil Del Alcazar CR, Aleckovic M, Polyak K: Immune Escape during Breast Tumor Progression. Cancer Immunol Res 2020, 8(4):422-427. 
21. Lazzeroni M, Dunn BK, Pruneri G, Jereczek-Fossa BA, Orecchia R, Bonanni B, DeCensi A: Adjuvant therapy in patients with ductal carcinoma in situ of the breast: The Pandora's box. Cancer Treat Rev 2017, 55:1-9.

22. Miligy I, Mohan P, Gaber A, Aleskandarany MA, Nolan CC, Diez-Rodriguez M, Mukherjee A, Chapman C, Ellis IO, Green AR et al: Prognostic significance of tumour infiltrating B lymphocytes in breast ductal carcinoma in situ. Histopathology 2017, 71(2):258268.

23. Chen XY, Thike AA, Nasir NDM, Koh VCY, Bay BH, Tan PH: Higher density of stromal M2 macrophages in breast ductal carcinoma in situ predicts recurrence. Virchows Archiv 2020, 476(6):825-833.

24. Thike AA, Koh V, Md Nasir ND, Tan PH: CD68 and CD163 predict recurrence and progression of breast ductal carcinoma in situ. Laboratory Investigation 2018, 98:108.

25. Campbell MJ, Baehner F, O’Meara T, Ojukwu E, Han B, Mukhtar R, Tandon V, Endicott M, Zhu Z, Wong J et al: Characterizing the immune microenvironment in high-risk ductal carcinoma in situ of the breast. Breast Cancer Research and Treatment 2017, 161(1):17-28.

26. Lotfınejad P, Kazemi T, Mokhtarzadeh A, Shanehbandi D, Jadidi Niaragh F, Safaei S, Asadi M, Baradaran B: PD-1/PD-L1 axis importance and tumor microenvironment immune cells. Life Sci 2020, 259:118297.

27. Adams S, Gatti-Mays ME, Kalinsky K, Korde LA, Sharon E, Amiri-Kordestani L, Bear H, McArthur HL, Frank E, Perlmutter J et al: Current Landscape of Immunotherapy in Breast Cancer: A Review. JAMA Oncol 2019, 5(8):1205-1214.

28. Yamashita N, Hisamatsu Y, Shigechi T, Tokunaga E, Saeki H, Oki E, Maehara Y: The immune microenvironment of ductal carcinoma in situ of the breast. Cancer Research 2019, 79(4).

29. Beguinot-Cornillon M, Dauplat MM, Kwiatkowski F, Lebouedec G, Tixier L, Pomel C, Penault-Llorca F, Radosevic-Robin N: Analysis of tumor-infiltrating lymphocytes (TILs) reveals biologically different subgroups of breast ductal carcinoma in situ. Cancer Research 2016, 76(14).

30. Bates GJ, Fox SB, Han C, Leek RD, Garcia JF, Harris AL, Banham AH: Quantification of regulatory T cells enables the identification of high-risk breast cancer patients and those at risk of late relapse. J Clin Oncol 2006, 24(34):5373-5380.

31. Dave R, Christie M, Parker B, Mann B: P143. Immune microenvironment as a prognostic marker of recurrence following breast conserving surgery for DCIS. European Journal of Surgical Oncology 2019, 45(5):921.

32. Agahozo MC, van Bockstal MR, Groenendijk FH, van den Bosch TPP, Westenend PJ, van Deurzen CHM: Ductal carcinoma in situ of the breast: immune cell composition according to subtype. Mod Pathol 2020, 33(2):196-205.

33. Thike AA, Chen XY, Koh VCY, Nasir N, Yeong JPS, Bay BH, Tan PH: Higher densities of tumour-infiltrating lymphocytes and CD4(+) T cells predict recurrence and progression of ductal carcinoma in situ of the breast. Histopathology 2020, 76(6):852-864.

\section{Tables}


Table 1. Characteristics of included studies in meta-analysis.

\begin{tabular}{|c|c|c|c|c|c|c|c|c|c|c|c|}
\hline Year & $\begin{array}{l}\text { First } \\
\text { author }\end{array}$ & Country & $\begin{array}{l}\text { Type of } \\
\text { DCIS }\end{array}$ & Cases & $\begin{array}{l}\text { TIL's } \\
\text { detection } \\
\text { method }\end{array}$ & $\begin{array}{l}\text { Cutoff of } \\
\text { TIL's } \\
\text { point }\end{array}$ & $\begin{array}{l}\text { Subtype } \\
\text { of TILs }\end{array}$ & Treatment & $\begin{array}{l}\text { Median } \\
\text { follow up }\end{array}$ & Deadline & Reference \\
\hline 2006 & $\begin{array}{l}\text { Gaynor J. } \\
\text { Bates et } \\
\text { al. }\end{array}$ & UK & p-DCIS & 62 & IHC & 15 & $\begin{array}{l}\text { FOXP3+ } \\
\text { TILs }\end{array}$ & $\begin{array}{l}\mathrm{S} \& \mathrm{AT} \\
(\mathrm{RT} / \mathrm{HT})\end{array}$ & $5.4 \mathrm{y}$ & $\mathrm{AR}$ & {$[30]$} \\
\hline 2016 & $\begin{array}{l}\text { G. Pruneri } \\
\text { et al. }\end{array}$ & Europe & DCIS & 1488 & $\mathrm{HE}$ & $\begin{array}{l}\text { Set1: } \\
<1 \% \text { vs. } \\
35 \%-50 \% \\
\text { Set2: } \\
<1 \% \text { vs. } \\
>50 \%\end{array}$ & $\begin{array}{l}\text { total p- } \\
\text { TILs }\end{array}$ & $\begin{array}{l}\mathrm{S} \& \mathrm{AT} \\
(\mathrm{RT} / \mathrm{HT})\end{array}$ & $8.2 \mathrm{y}$ & IR & {$[8]$} \\
\hline 2015 & $\begin{array}{l}\text { Elizabeth } \\
\text { Thompson } \\
\text { et al. }\end{array}$ & USA & $\begin{array}{l}\text { p-DCIS } \\
\& \\
\text { i-DCIS }\end{array}$ & 27 & IHC & $50 \%$ & $\begin{array}{l}\text { PD-L+ } \\
\text { TILs }\end{array}$ & $\mathrm{S} \& \mathrm{AT}$ & $80 \mathrm{~m}$ & IR & [9] \\
\hline 2017 & $\begin{array}{l}\text { Shona } \\
\text { Hendry et } \\
\text { al. }\end{array}$ & Australia & p-DCIS & $\begin{array}{l}\text { HE: (n } \\
=138) \\
\text { IHC: }(\mathrm{n} \\
=79)\end{array}$ & $\begin{array}{l}\mathrm{HE} \& \\
\mathrm{IHC}\end{array}$ & $\begin{array}{l}\text { TILs: } \% \text {. } \\
\text { PD-L1: } \\
1 \%\end{array}$ & $\begin{array}{l}\text { total p- } \\
\text { TILs } \\
\text { PD-L1+ } \\
\text { TILs } \\
\text { PD-L1+ } \\
\text { TC }\end{array}$ & $\begin{array}{l}\text { S \& AT } \\
(\mathrm{RT} / \mathrm{HT})\end{array}$ & $\begin{array}{l}\text { TILs :89 } \\
\text { m } \\
\quad \text { PD- } \\
\text { L1:39 m }\end{array}$ & IR & [7] \\
\hline \multirow[t]{2}{*}{2018} & \multirow[t]{2}{*}{$\begin{array}{l}\text { Michael S. } \\
\text { Toss et al. }\end{array}$} & \multirow[t]{2}{*}{ UK } & $\begin{array}{l}\text { training } \\
\text { set: } \\
\text { p-DCIS }\end{array}$ & $\begin{array}{l}\text { training } \\
\text { set: } \\
(n=150)\end{array}$ & \multirow[t]{2}{*}{$\mathrm{HE}$} & $\begin{array}{l}\text { training } \\
\text { set: } 5 \%\end{array}$ & $\begin{array}{l}\text { training } \\
\text { set: } \\
\text { total p- } \\
\text { TILs }\end{array}$ & $\begin{array}{l}\text { training } \\
\text { set: } \\
\text { S/ S \& RT }\end{array}$ & $\begin{array}{l}\text { training } \\
\text { set: } 161 \mathrm{~m}\end{array}$ & \multirow[t]{2}{*}{ IR } & \multirow[t]{2}{*}[14]{} \\
\hline & & & $\begin{array}{l}\text { validation } \\
\text { set: } \\
\text { p-DCIS }\end{array}$ & $\begin{array}{l}\text { validation } \\
\text { set: } \\
(\mathrm{n}=534)\end{array}$ & & $\begin{array}{l}\text { validation } \\
\text { set: } 20\end{array}$ & $\begin{array}{l}\text { validation } \\
\text { set: } \\
\text { total t- } \\
\text { TILs }\end{array}$ & $\begin{array}{l}\text { validation } \\
\text { set: } \\
\text { BCS }\end{array}$ & $\begin{array}{l}\text { validation } \\
\text { set: } 109 \\
\text { m }\end{array}$ & & \\
\hline 2019 & $\begin{array}{l}\text { Rajiv Dave } \\
\text { et al. }\end{array}$ & Australia & p-DCIS & 423 & $\mathrm{HE}$ & $5 \%$ & $\begin{array}{l}\text { total p- } \\
\text { TILs }\end{array}$ & $\mathrm{BCS}$ & $119 \mathrm{~m}$ & IR & {$[31]$} \\
\hline 2020 & $\begin{array}{l}\text { Marie } \\
\text { Colombe } \\
\text { Agehozo } \\
\text { et al. }\end{array}$ & Netherlands & p-DCIS & $\begin{array}{l}\text { HE: } \\
(n=466) \\
\text { IHC: } \\
(n=115 \square\end{array}$ & $\begin{array}{l}\text { HE \& } \\
\text { IHC }\end{array}$ & $30 \%$ & $\begin{array}{l}\text { total p- } \\
\text { TILs } \\
\text { PD-L1+ } \\
\text { TILs } \\
\text { PD-L1+ } \\
\text { TC }\end{array}$ & BCS/ MAS & $98 \mathrm{~m}$ & IR & {$[32]$} \\
\hline 2019 & $\begin{array}{l}\text { Farbod } \\
\text { Darvishian } \\
\text { et al. }\end{array}$ & USA & p-DCIS & 69 & $\mathrm{HE}$ & $45 \%$ & $\begin{array}{l}\text { total p- } \\
\text { TILs }\end{array}$ & BCS & $6.7 \mathrm{y}$ & IR & [11] \\
\hline 2019 & $\begin{array}{l}\text { Mieke Van } \\
\text { Bockstal } \\
\text { et al. }\end{array}$ & Belgium & p-DCIS & 211 & $\mathrm{HE}$ & $50 \%$ & $\begin{array}{l}\text { total p- } \\
\text { TILs }\end{array}$ & $\begin{array}{l}\mathrm{S} \& \mathrm{AT} \\
(\mathrm{RT} / \mathrm{HT})\end{array}$ & $124 \mathrm{~m}$ & IR & [10] \\
\hline 2020 & $\begin{array}{l}\text { Aye Aye } \\
\text { THIKE et } \\
\text { al. }\end{array}$ & Singapore & DCIS & 198 & $\begin{array}{l}\text { HE \& } \\
\text { IHC }\end{array}$ & $20 \%$ & $\begin{array}{l}\text { total p- } \\
\text { TILs } \\
\text { CD4+ } \\
\text { TILs }\end{array}$ & $\begin{array}{l}\text { S (BCS/ } \\
\text { MAS) }\end{array}$ & $\begin{array}{l}\text { TILs: } 7.9 \\
\text { y } \\
\text { CD } 4+: \\
7.2 \text { y }\end{array}$ & $\mathrm{AR}$ & [33] \\
\hline 2020 & $\begin{array}{l}\text { Michael S. } \\
\text { Toss et al. }\end{array}$ & UK & p-DCIS & 508 & $\begin{array}{l}\text { HE \& } \\
\text { IHC }\end{array}$ & 20 & $\begin{array}{l}\text { total t- } \\
\text { TILs } \\
\text { FOXP3+ }\end{array}$ & BCS & unknown & IR & [12] \\
\hline
\end{tabular}




\begin{tabular}{|c|c|c|c|c|c|c|c|c|c|c|c|}
\hline & & & & & & & $\begin{array}{l}\text { TILs } \\
\text { PD-L1+ } \\
\text { TILs } \\
\text { CD4+ } \\
\text { TILs } \\
\text { CD8+ } \\
\text { TILs } \\
\text { PD-L1+ } \\
\text { TILs }\end{array}$ & & & & \\
\hline 2020 & $\begin{array}{l}\text { Alberto } \\
\text { Farolfi et } \\
\text { al. }\end{array}$ & Italy & p-DCIS & 496 & $\mathrm{HE}$ & $5 \%$ & $\begin{array}{l}\text { total p- } \\
\text { TILs }\end{array}$ & $\mathrm{S} / \mathrm{S} \& \mathrm{RT}$ & $56.4 \mathrm{y}$ & SBE & [15] \\
\hline 2021 & $\begin{array}{l}\text { Fei-Fei Xu } \\
\text { et al. }\end{array}$ & China & $\begin{array}{l}\text { p-DCIS } \\
\& \\
\text { m-DCIS }\end{array}$ & 135 & $\mathrm{HE}$ & 5 & $\begin{array}{l}\text { total t- } \\
\text { TILs }\end{array}$ & $\begin{array}{l}\text { BCS/ BCS } \\
\& \mathrm{RT}\end{array}$ & $53 \mathrm{~m}$ & IR & [13] \\
\hline
\end{tabular}

Note: UK (United Kingdom); USA (United States of America); p-DCIS (pure DCIS); i-DCIS (pure DCIS mixed with IDC); m-DCIS (pure DCIS mixed with microinvasive cancer); HE (hematoxylin and eosin staining); IHC (Immunohistochemistry staining); TILs (tumor-infiltrating lymphocytes); t-TILs (stromal touching TILs); p-TILs (percentage of stromal TILs); TC (tumor cell); S (surgery); BCS (breast conserving surgery); MAS (mastectomy); AT (adjuvant therapy); RT (radiation therapy); HT (hormonal therapy); IR (ipsilateral recurrence); AR (all kinds of recurrence); SBE (second breast cancer events) 
Table 2: Summary of the prognostic value of TILs obtained from the subgroup analysis including dense TIL's assessment, Deadline, Variable, Location of TILs, Cutoff of TILs, Therapeutic approach, Pathology and subsets of TILs

\begin{tabular}{|c|c|c|c|c|c|c|c|}
\hline \multirow[t]{2}{*}{ Subgroup } & \multirow[t]{2}{*}{$\mathbf{N}$} & \multicolumn{2}{|c|}{ Random-effects model } & \multicolumn{2}{|l|}{ Fixed-effects model } & \multicolumn{2}{|c|}{ Heterogeneity } \\
\hline & & HR $(95 \% \mathrm{CI})$ & $P$ & HR $(95 \% \mathrm{CI})$ & $P$ & $I 2$ & $P h$ \\
\hline Dense TIL's assessment & 14 & $2.11(1.35-3.28)$ & 0.001 & $2.13(1.77-2.55)$ & 0.001 & $78.30 \%$ & 0 \\
\hline percentage of stromal TILs & 11 & $1.49(1.11-1.99)$ & 0.008 & $1.45(1.16-1.80)$ & 0 & $28.40 \%$ & 0.175 \\
\hline number of touching TILs & 3 & $4.73(2.28-9.80)$ & 0 & $5.1(3.66-7.11)$ & 0 & $72.40 \%$ & 0.027 \\
\hline Deadline & 14 & $2.11(1.35-3.28)$ & 0.001 & $2.13(1.77-2.55)$ & 0 & $78.30 \%$ & 0 \\
\hline ipsilateral recurrence & 12 & $2.26(1.31-3.91)$ & 0.004 & $2.33(1.89-2.88)$ & 0 & $80.60 \%$ & 0 \\
\hline all kinds of recurrence & 2 & $1.61(1.11-2.33)$ & 0.011 & $1.61(1.11-2.33)$ & 0.011 & 0 & 0.662 \\
\hline Variable & 14 & $2.11(1.35-3.28)$ & 0.001 & $2.13(1.77-2.55)$ & 0 & $78.30 \%$ & 0 \\
\hline univariate analysis & 5 & $1.42(1.02-1.99)$ & 0.039 & $1.42(1.02-1.99)$ & 0.039 & $0.00 \%$ & 0.42 \\
\hline multivariate analysis & 9 & $2.30(1.27-4.17)$ & 0.006 & $2.52(2.03-3.14)$ & 0 & $83.40 \%$ & 0 \\
\hline Location of TILs & 14 & $2.11(1.35-3.28)$ & 0.001 & $2.13(1.77-2.55)$ & 0 & $78.30 \%$ & 0 \\
\hline touching TILs & 2 & $6.04(2.64-13.81)$ & 0 & $6.04(2.64-13.81)$ & 0 & 0 & 0.958 \\
\hline stromal TILs & 12 & $1.81(1.13-2.90)$ & 0.013 & $2.02(1.67-2.43)$ & 0 & $79.40 \%$ & 0 \\
\hline Cutoff of TILs & 14 & $2.11(1.35-3.28)$ & 0.001 & $2.13(1.77-2.55)$ & 0 & $78.30 \%$ & 0 \\
\hline $1 \%-5 \%$ & 6 & $1.24(0.90-1.70)$ & 0.186 & $1.24(0.90-1.70)$ & 0.186 & 0 & 0.933 \\
\hline $6 \%-30 \%$ & 2 & $1.73(1.17-2.56)$ & 0.006 & $1.73(1.17-2.56)$ & 0.006 & 0 & 0.69 \\
\hline $31 \%-50 \%$ & 3 & $3.03(0.71-12.97)$ & 0.134 & $1.57(0.97-2.56)$ & 0.068 & $81.20 \%$ & 0.005 \\
\hline counts ( 5 or 15 or 20 ) & 3 & $4.73(2.28-9.80)$ & 0 & $5.10(3.66-7.11)$ & 0 & $72.40 \%$ & 0.027 \\
\hline Therapeutic approach & 14 & $2.11(1.35-3.28)$ & 0.001 & $2.13(1.77-2.55)$ & 0 & $78.30 \%$ & 0 \\
\hline breast conserving therapy or mastectomy & 5 & $2.77(1.26-6.07)$ & 0.011 & $2.51(1.99-3.17)$ & 0 & $89.50 \%$ & 0 \\
\hline breast conserving therapy + radiotherapy & 4 & $2.26(1.29-3.95)$ & 0.004 & $2.24(1.49-3.35)$ & 0 & $42.10 \%$ & 0.159 \\
\hline $\begin{array}{l}\text { surgery + adjuvant therapies } \\
\text { (hormonal therapy, radiotherapy) }\end{array}$ & 5 & $1.16(0.62-2.18)$ & 0.645 & $1.07(0.69-1.68)$ & 0.753 & $28.30 \%$ & 0.233 \\
\hline Pathology & 14 & $2.11(1.35-3.28)$ & 0.001 & $2.13(1.77-2.55)$ & 0 & $78.30 \%$ & 0 \\
\hline pure DCIS & 9 & $2.37(1.31-4.28)$ & 0.004 & $2.54(2.02-3.18)$ & 0 & $81.90 \%$ & 0 \\
\hline $\begin{array}{l}\text { pure DCIS \& } \\
\text { pure DCIS mixed with invasive cancer }\end{array}$ & 2 & $5.03(1.63-15.52)$ & 0.005 & $5.09(1.71-15.13)$ & 0.003 & $1.60 \%$ & 0.313 \\
\hline DCIS & 3 & $1.32(0.88-1.97)$ & 0.184 & $1.36(0.98-1.89)$ & 0.064 & $22.60 \%$ & 0.275 \\
\hline subset of TILs & 10 & $2.29(1.31-3.99)$ & 0.003 & $2.55(2.06-3.16)$ & 0 & $77.80 \%$ & 0 \\
\hline CD4+ TILs & 2 & $1.98(1.14-3.44)$ & 0.015 & $1.97(1.31-2.96)$ & 0.001 & $45.80 \%$ & 0.174 \\
\hline CD8+ TILs & 1 & $0.90(0.47-1.71)$ & 0.747 & $0.90(0.47-1.71)$ & 0.747 & $0.00 \%$ & - \\
\hline FOXP3+ TILs & 2 & $1.83(1.23-2.70)$ & 0.003 & $1.83(1.23-2.70)$ & 0.003 & $0.00 \%$ & 0.382 \\
\hline PD-L1+ TILs & 5 & $6.21(4.26-9.06)$ & 0 & $6.21(4.26-9.06)$ & 0 & $0.00 \%$ & 0.708 \\
\hline
\end{tabular}




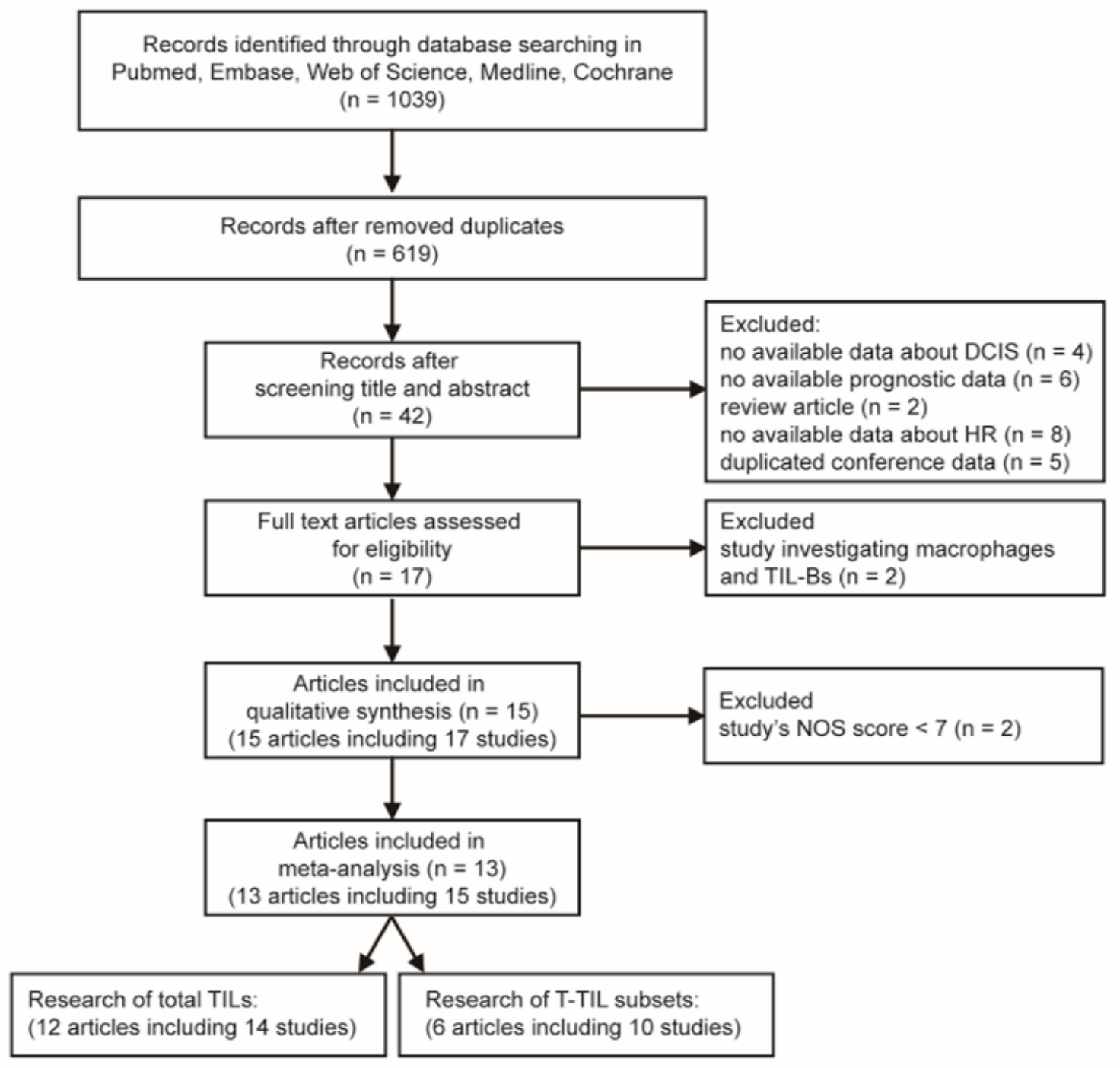

\section{Figure 1}

Flow diagram of study selection and identification. 
A

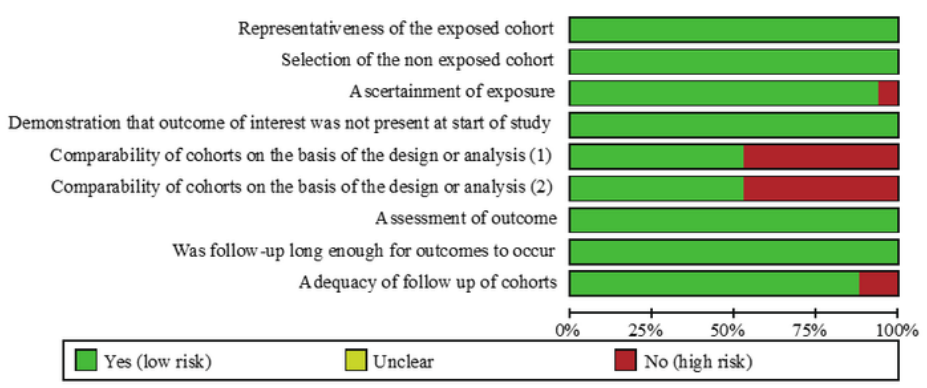

B

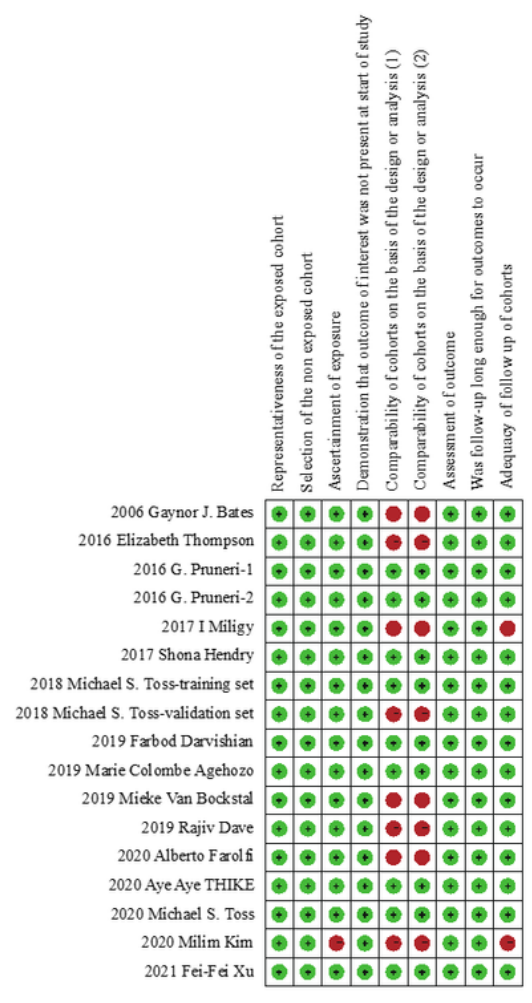

\section{Figure 2}

Risk of bias assessment of included studies. A. Risk of bias graph: review authors' judgments about each risk of bias item presented as percentages across all included studies; B. Risk of bias summary: review authors' judgments about each risk of bias item for each included study. 
A

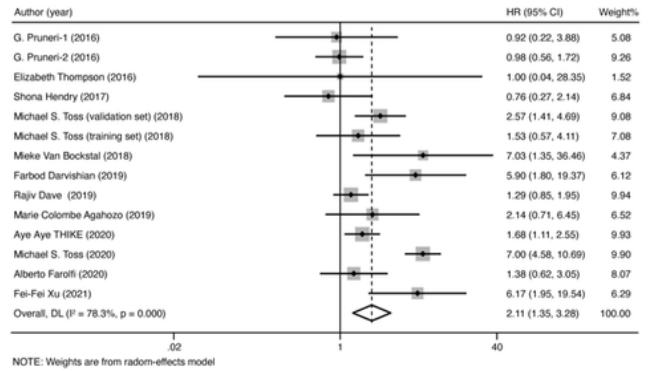

B

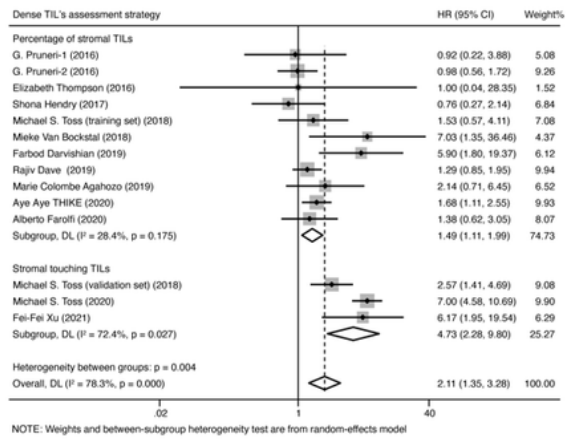

C

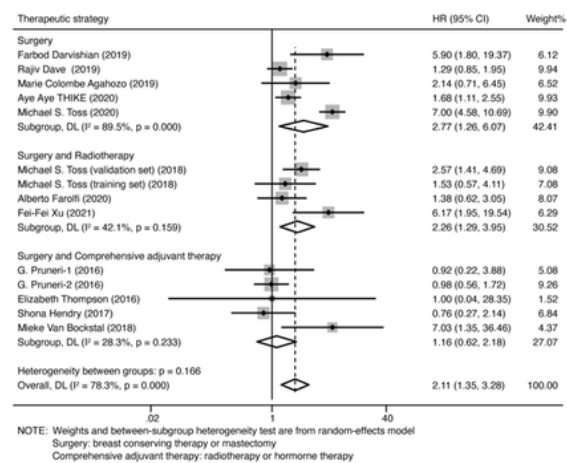

\section{Figure 3}

Forest plots of the prognostic value of TILs in patients diagnosed with DCIS. A. Forest plots of prognostic value of total TILs in DCIS; B. Forest plots of prognostic value of TILs assessed with different strategies on recurrence risk of DCIS; C. Forest plots of prognostic value of TILs on patients who receiving different therapies. 
A

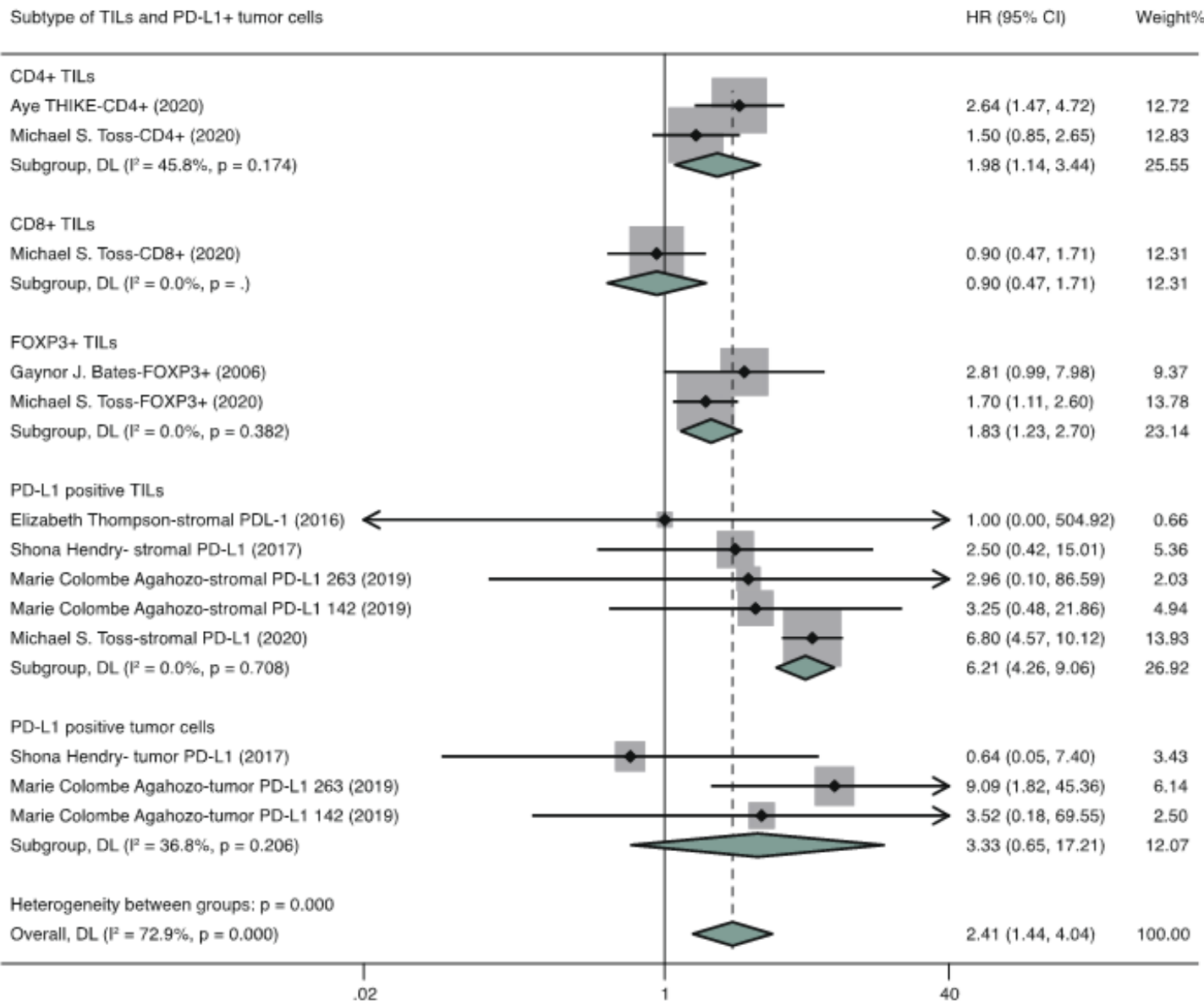

NOTE: Weights and between-subgroup heterogeneity test are from random-effects model

\section{Figure 4}

Forest plots of the prognostic value of different subsets of T-TILs and PD-L1+ tumor cells in DICS. 
A

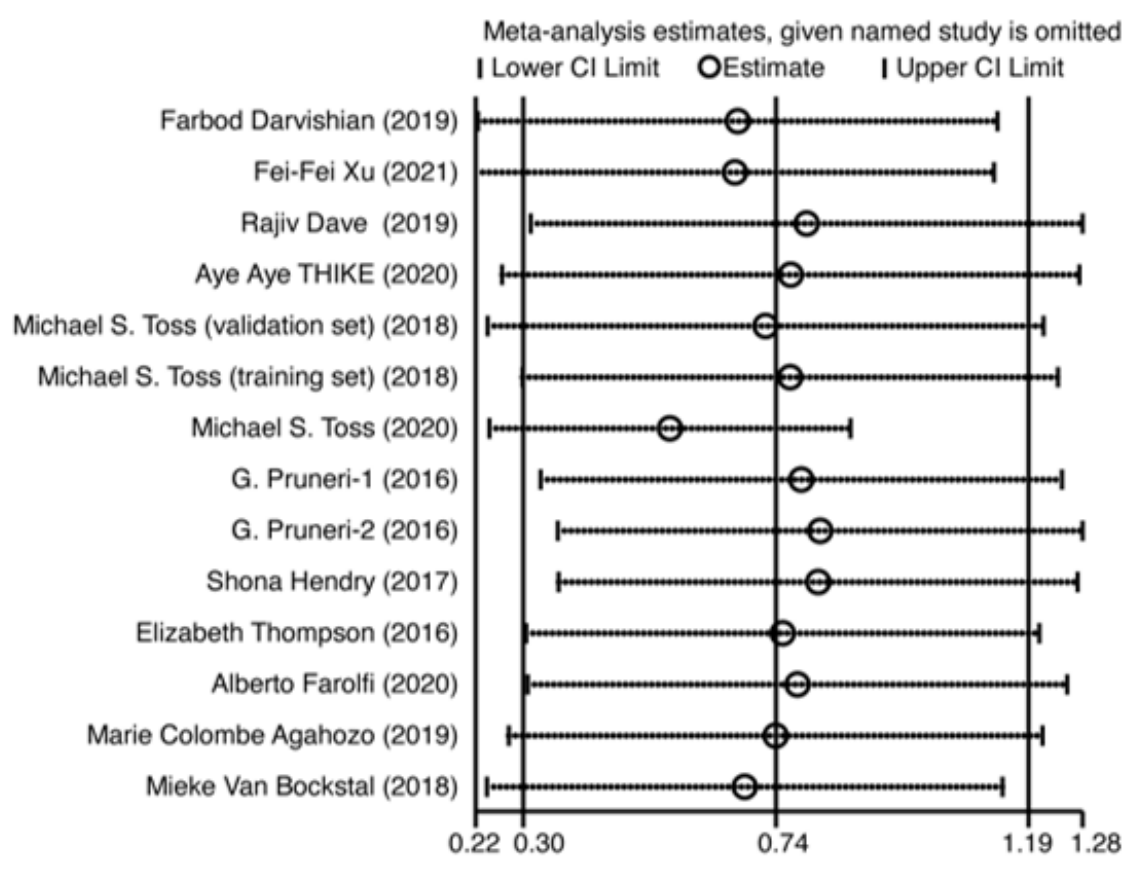

Figure 5

Sensitivity analysis of the meta-analysis of total TILs in DCIS. 
A

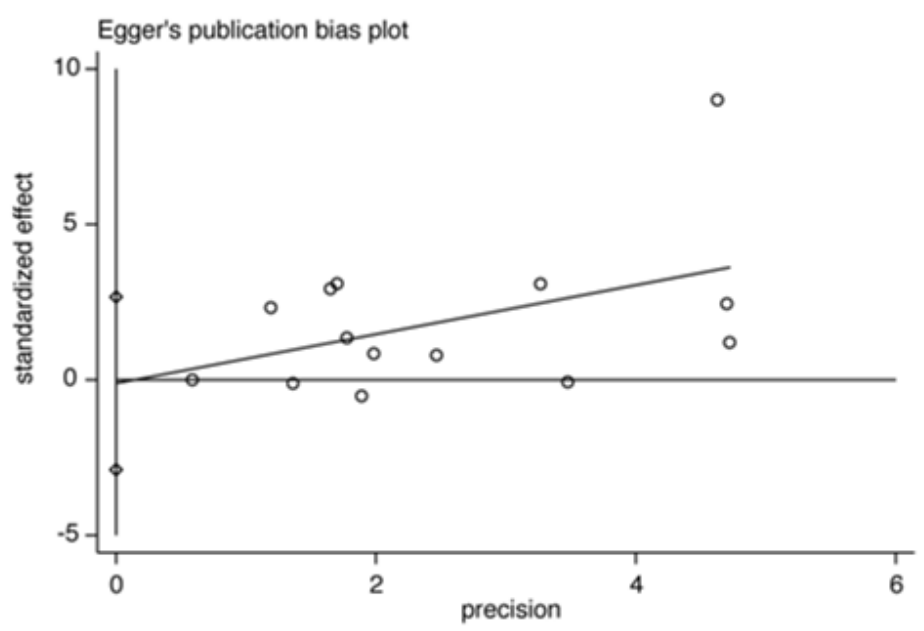

B

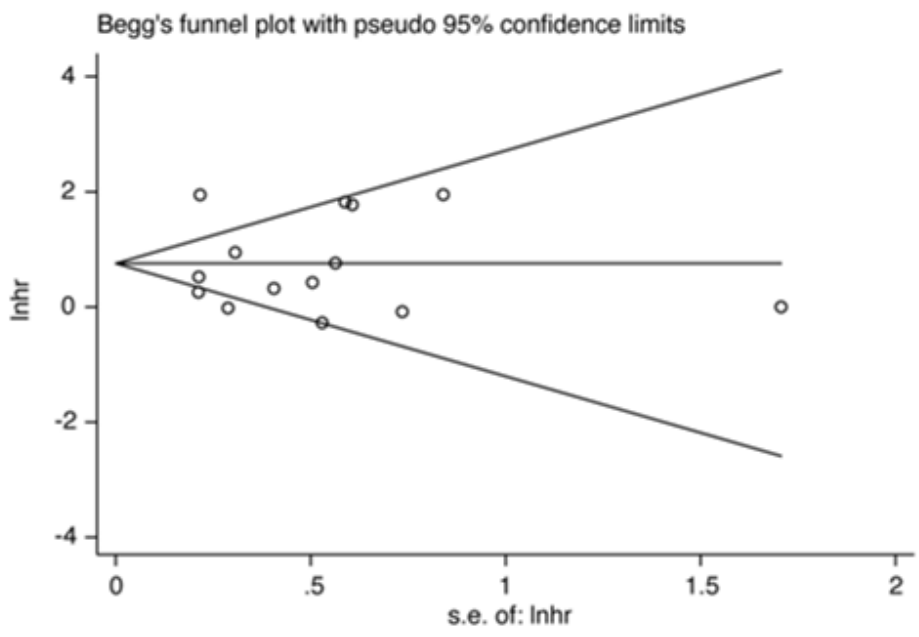

Figure 6

Funnel plots of potential publication bias with Egger's test (A) and Begg's test (B). 